\title{
Alexander Rauhut* Exploring the Effect of Conversion on the Distribution of Inflectional Suffixes: A Multivariate Corpus Study
}

https://doi.org/10.1515/zaa-2021-2024

Abstract: Lexical ambiguity in the English language is abundant. Word-class ambiguity is even inherently tied to the productive process of conversion. Most lexemes are rather flexible when it comes to word class, which is facilitated by the minimal morphology that English has preserved. This study takes a multivariate quantitative approach to examine potential patterns that arise in a lexicon where verb-noun and noun-verb conversion are pervasive. The distributions of three inflectional suffixes, verbal -s, nominal -s, and -ed are explored for their interaction with degrees of verb-noun conversion. In order to achieve that, the lexical dispersion, context-dependency, and lexical similarity between the inflected and bare forms were taken into consideration and controlled for in a Generalized Additive Models for Location, Scale and Shape (GAMLSS; Stasinopoulos, M. D., R. A. Rigby, and F. De Bastiani. 2018. "GAMLSS: A Distributional Regression Approach.” Statistical Modelling 18 (3-4): 248-73). The results of a series of zero-one-inflated beta models suggest that there is a clear "uncanny" valley of lexemes that show similar proportions of verbal and nominal uses. Such lexemes have a lower proportion of inflectional uses when textual dispersion and context-dependency are controlled for. Furthermore, as soon as there is some degree of conversion, the probability that a lexeme is always encountered without inflection sharply rises. Disambiguation by means of inflection is unlikely to play a uniform role depending on the inflectional distribution of a lexeme.

Keywords: conversion, dispersion, gamlss regression, lexical ambiguity, multivariate, verb-noun continuum

\section{Introduction}

The English language makes it easy to verb a noun. Conversion is a remarkably productive word formation process in analytic languages. Yet little quantitative

*Corresponding author: Alexander Rauhut, Freie Universität Berlin, Institut für Englische Philologie, Habelschwerdter Allee 45, 14195 Berlin, Germany, E-mail: alexander.rauhut@fuberlin.de 
research has been presented on the topic. This study will focus on word class ambiguity and explore its effect on the distribution of inflections. I will attempt to show that the probability of occurrence of inflectional suffixes is affected by the relative frequency of nominal versus verbal uses, textual specificity, as well as lexical and contextual boundedness. Forms that show a similar degree of association to both categories exist in a potential "uncanny valley", and it will be argued that there is a noticeably different distribution of inflectional suffixes for those forms. These differences might point to systemic tendencies or even restrictions that, among other reasons, could be caused by a more general tendency to avoid ambiguity.

Ambiguity is pervasive in language. In fact, the term itself is ambiguous and there is a plethora of types of ambiguity that have been recognized by linguists. On the structural level of English, ambiguities can be roughly categorized into two broad categories of syntactic, and lexical ambiguities. Lexical ambiguity most frequently occurs when there is polysemy or homonymy that may lead to conflicting interpretations. Syntactic ambiguity arises when there are multiple parses for the same syntactic pattern. This can itself be directly or indirectly caused by some form of lexical ambiguity. Word class ambiguity is a type of ambiguity that is not so commonly in focus. There is little potential for misinterpreting conversion or accidentally assuming conversion. Most lexemes that are commonly categorized as belonging to one of the open word classes are ambiguous with respect to word class only outside of a communicative context. Word-class information and other grammatical and distributional information is stored in memory as part of the information about the lexeme, so this ambiguity is inherently a matter of degree. Lexical items are more or less strongly entrenched. How word-class ambiguous a lexeme is also depends on the distributional make-up and semantic properties. The aim of this study is not to identify and analyze actual cases of ambiguity that are caused by conversion, but rather to investigate large scale effects and tendencies on the composition of the lexical system. The underlying question is why a language like English allows speakers to readily use a lexical item as either noun or verb even though there is little morphological marking in English.

The emergence of lexical clusters, including polysemes, homonyms or multiword expressions is conditioned by frequency. In an exemplar-based model, word classes themselves are expected to emerge through categorizing and clustering of contiguous experiences, e.g. (co-)occurrence patterns. Various studies have provided evidence for frequency and clustering effects (for a review, cf. Diessel 2016). Conversely, the lack of distinctiveness between (co-)occurrence patterns is expected to cause fuzzier, generally less well-entrenched categories. If conversion becomes too frequent, the clustering of occurrences per lexeme and the learned associations to the word classes would be weakened for the affected items. 
In Sections 2.1 and 2.2, I will outline the phenomenon and review experimental phonetics and neurolinguistics literature on the effects of ambiguity and ambiguity avoidance, and discuss some arguments on the role and limits of ambiguity. Section 2.3 is concerned with the conceptualization and operationalization of conversion, and Section 3 will provide an overview over the metrics used in this study. Subsequently, in Section 4, I will present a series of GAMLSS models (Generalized Additive Models for Location, Scale and Shape, cf. Stasinopoulos, Rigby, and Bastiani 2018; Rigby and Stasinopoulos 2005) that attempt to model the influence of verb-noun or noun-verb conversion on the distribution of -s and -ed suffixation while controlling for textual dispersion, regularity of occurrence and contextual flexibility. In Section 5, the results will be discussed in light of considerations from previous sections. Section 6 concludes the findings and provides suggestions for improvements in methodology. ${ }^{1}$

\section{Word Class Ambiguity}

\subsection{Potential Functions of Ambiguity}

Wasow (2015) provides an overview of different types of ambiguities. Among other types, the author points out that word order freezing might be seen as ambiguity avoidance. The ambiguity that is hypothesized to be avoided here is understood to be concerning syntactic relations. However, word order freezing also avoids word class ambiguity in a mostly inflection-free grammar. German has a rather free word order, but the finite verb is also frozen in second position, which equally avoids word class ambiguity, especially considering German bare infinitives are a common means of nominalization. Wasow (2015) finally concludes that ambiguity avoidance has not been shown to be as common as expected considering the pervasiveness of ambiguities in language.

Phonetic changes are often the trigger for the emergence of homonymy and some types of syntactic ambiguity, mostly those caused by syncretism via morphological reduction. Evidently, those ambiguities are often not immediately resolved or compensated for in an obvious way. Past research has tried to explain the existence and even necessity of ambiguity in natural languages. There seem to be good reasons for the pervasiveness of ambiguity, and good reasons for a language to keep ambiguity at a minimum. In both cases, ease of processing and

1 The data was preprocessed using the Corpus Workbench (Evert and Hardie 2011), all analyses were coded in $R$ 4.1.0 (Dowle and Srinivasan 2021; R Core Team 2021), figures were created with ggplot2 (Wickham 2016). 
ease of production represent convincing candidates (cf. Piantadosi, Tily, and Gibson 2012; Tomaschek et al. 2018). They are also competing motivations potentially balancing the amount of ambiguity in a language. Piantadosi, Tily, and Gibson (2012) argue that some degree of ambiguity is required in an efficient communicative system. More recently, Piantadosi, Tily, and Gibson (2012) argued that, based on simulation, linguistic forms are expected to show a certain degree of ambiguity in order to provide an efficient system. Their simulations suggest that ambiguity caused by homophony is more common in natural languages than expected, despite the processing and efficiency advantages. Furthermore, homophones were shown to be smoothed out in lexically neighboring areas.

Given the plethora of contextual information in any given communicative situation, a different reason for the perceived pervasiveness of ambiguity is that there is usually enough context to disambiguate. Studies like Plag, Homann, and Kunter (2017) on word-final -s call into question how common perfect homophony actually is. The study found significant length differences, and hypothesizes that subtle phonetic differences are learned and contribute to the lexical representation of words in memory. Systematic phonetic differences potentially contribute to disambiguation. Yung Song et al. (2013), in a more lexically restricted study, did not find any such effect. In a more recent study, Tomaschek et al. (2021) show that length of final - $s$ can be modeled as having a discriminatory function depending on the lexical and phonological context. In other words, the duration was found to decrease with increasing contextual ambiguity. "Energy is not invested in a signal that creates confusion instead of clarity." (Tomaschek et al. 2021: 154) This points to some degree of ambiguity sensitivity, even though it is not entirely clear what it means for ambiguity avoidance. Assuming the inverse of this observation is equally the case, namely that more energy is invested in a signal that creates clarity, an interesting analogical hypothesis could be formulated that the word class of a word-class ambiguous lexeme is potentially reinforced by additional morpho-syntactic marking even though they are not necessarily likely to produce ambiguity. In that case, redundancies in marking are to be expected.

\subsection{Word Class Ambiguity and Processing}

In neurolinguistic experiments it has been observed that word class ambiguity leads to significantly longer processing times of word-class ambiguous items (Federmeier et al. 2000; Lee and Federmeier 2008). This correlates with the activation of separate regions in memory for nominal and verbal representations of the item. Interestingly, these effects are present even when word class membership is clear from the context, although these findings have been somewhat relativized by 
Lee and Federmeier (2006), where it is argued that other types of semantic ambiguity are also common in many of the word class ambiguous items, which might cause part of the effect. Nevertheless, the idea that syntactic contexts that allow hearers to disambiguate the word class of an item may still not be enough for optimizing processing time would support the hypothesized need for redundancy mentioned above. In contrast to the increased difficulty in processing, and in accordance with the idea of competing motivations, Bultena, Dijkstra, and Hell (2013) describe a facilitatory effect of word class ambiguity for learners.

In order to investigate how word class ambiguity affects the distribution of the available inflectional markers, the next section will focus on the frequency distribution of word class per lexical item in a corpus.

\subsection{Word Class Ambiguity in Corpora}

The potential for conversion and word class ambiguity varies from lexeme to lexeme. Conversion can be spontaneous or lexicalized, showing different degrees of conventionalization. It can be assumed that there is a cline allowing for all degrees of lexicalization and conventionalization from polysemy up to full-fledged homonymy with varying degrees of lexical association between the nominal and verbal counterparts.

(1) spontaneous: streisanded (twitter hashtag ${ }^{2}$ )

(2) lexicalized: a build, to build

(3) partial homonymy: a form, to form

(4) full homonymy: a bank, to bank

Conversion is a productive process that inevitably produces homophones. Additionally, noun-verb and verb-noun conversions are doubly homophonous with inflectional affixes in $-s$.

In order to express the proportions of nominal and verbal tagging, the ratio of the less dominant word class to the more dominant word class was taken per lemma. When a lemma had a dominantly verbal use, the value was subtracted from 1, while 1 was subtracted from the value for dominantly nominal uses. This puts the measure into the range $[-1,1]$ with -1 when the item was always tagged as verb, +1 when it was always tagged as noun and 0 when there was a perfect

2 https://twitter.com/hashtag/streisanded. 

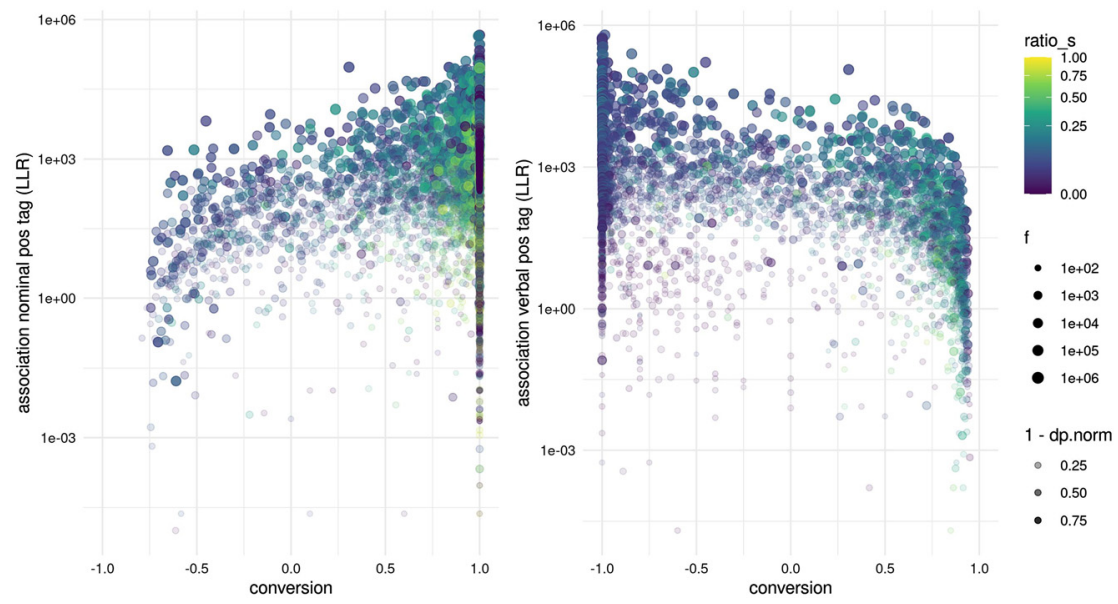

Figure 1: Compound ratio of noun/verb POS tags against attraction.

balance. Figure 1 shows the statistical attraction (measured by the log-likelihood ratio (cf. Evert 2005)) to nominal and verbal uses plotted against the mentioned conversion ratio. Note that lemmas that exhibit negative statistical association to the respective POS tag are omitted in the plot. The measure appears to connect the two distributions well, resulting in a rather homogenous continuum across which the attraction to nominal/verbal uses increases monotonously. The "uncanny valley" at around 0 is less densely populated, which is expected since there should be discretization effects between the word classes considering afore-mentioned clustering effects.

\section{Methodology}

\subsection{Textual Dispersion}

In order to measure the textual specificity of lexemes, and account for lexical items with very specific contextually bound uses, two types of dispersion are used. The first is the Deviation of Proportions across corpus parts (DP, Gries 2008), more specifically the normalized version (DP.norm, Lijffijt and Gries 2012). As the basic unit, the individual texts were used based on text ID. DP.norm is a corpus-partbased measure bound between 0 and 1 . Values close to 1 indicate a high deviation, therefore a low dispersion. It can be interpreted as a measure of how evenly tokens are spread over the corpus parts. Figure 1 demonstrates how DP can be used for 
explorative visualization, e.g. by using it to scale alpha values in dense overplotted scatterplots.

$D P$ cannot account for short bursts of occurrences, therefore, Word Growth Dispersion (DWG) (Zimmermann 2020) is used in addition, which is a wholecorpus measure based on distances between occurrences. $D W G$ is a measure of how regularly a token occurs across an entire corpus occurs across an entire corpus. A geometric normalization is applied to account for sample size (cf. Zimmermann 2020). It is a measure bound between 0 and 1 with higher values indicating higher dispersion. Both measures were designed to measure dispersion or commonness of lexical items as extension to the most commonly used plain frequencies. The two measures highlight different aspects both conceptually and empirically (cf. Gries 2021 on using multiple measures of dispersion and association). Therefore, both correlate with frequency $(f)$ of use, even though $D W G$ does so to a lesser degree.

Based on observations from previous sections, it is expected that in the presence of word class ambiguity, a lower context-dependency/lower clumpiness indirectly leads to higher proportions of inflectional uses since non-inflectional uses would be more ambiguous and harder to process. Considering a given dispersion across contexts in which a lexeme is likely to occur, the existence of avoidance contexts should manifest in a penalty to dispersion and make the distribution clumpier. It is difficult to formally identify avoidance contexts. Lexical and syntactic correlates of the avoided structure might be avoided as a side effect, and cause fuzzier overall differences in structure. The contexts in which ambiguity is avoided/not avoided might be rather evenly dispersed themselves. This could mask potential clumpiness of -s occurrences, adding additional noise. Effects that influence dispersion might be washed out because of that. Nevertheless, both measures allow us to control for higher than usual frequencies of observations caused by repetition in rapid succession or concentrated in few texts.

Both extremely high and extremely low values of DWG and DP.norm suggest special values. Most of the variables used in the model are not expected to have a monotonic relationship with the proportion of -s occurrences. Very unevenly dispersed lexemes are overrepresented in terms of frequency, and an extremely even dispersion suggests uses typical of function words.

\subsection{Fixedness}

The ratio of hapaxes (henceforth $a l p h a_{1}$, Evert 2005: 130) on either side of a given lexeme was used as a simple measure of how fixed its immediate lexicogrammatical context is. The term hapax is used a liberally here, referring to types 
that occur only once within a given window. For ease of interpretation and in order to capture the fixedness in smaller units of text, this window had a size of 1 token to the left and right (preliminary experiments with larger windows were inconclusive, so the simplest version was used). The left and right contexts were also kept separate since lumping them together conflates quite different pieces of information, considering the direction of processing, the branching structure of English, priming effects etc. For example, a token that is always preceded by a definite article as a part of a name scores an extremely low alpha $a_{1}$ value.

Lemmas that had $-s$ forms that only occurred in totally fixed contexts, therefore scoring alph $_{1}$ values of lower than 0.3 , were excluded as outliers. The exclusion of these observations slightly improved fit of the models. On the lower frequency bands this is due to a substantial decrease of noise as it is difficult to estimate the proportion of $-s$ only based on a few occurrences of $-s$. For the higher frequency bands, examples for such outliers include abbreviations (e.g. e.g., pp., etc., etc.), and parts of multi-word names (e.g. New Zealander). The latter type of exclusion is operationally consistent with the focus on single-word units (see Section 4.2). Some multi-word structures should be treated as individual units and as a distinct lexeme, but this is beyond the scope of this study.

Similarly to what has been observed for dispersion measures, both extremely high and extremely low values are untypical. For example, a noun that has an extremely high hapax ratio in its left context is unlikely to be preceded by determiners, which is unusual for a noun.

\subsection{Cosine Similarities of Word Vectors}

As a final measure to explore, I trained a simple GloVe embedding (Pennington, Socher, and Manning 2014; Selivanov, Bickel, and Wang 2020) on the dataset. The resulting word vectors are a numerical representation of a two-layer neural network reconstructing the co-occurrence patterns of each lemma. They have been shown to capture lexical and semantic information rather well. From the trained word vectors, I obtained the cosine similarities between the base forms and affixed forms which will be used as another co-occurrence metric in the final models.

The color shade in Figure 2 shows that the cosine similarity between base and inflected form appears to be correlated with a higher frequency of inflected forms. Part of the reason is that it is weakly correlated with frequency. The variability lies in the fact that some degree inflection, especially for plural -s, might change the 

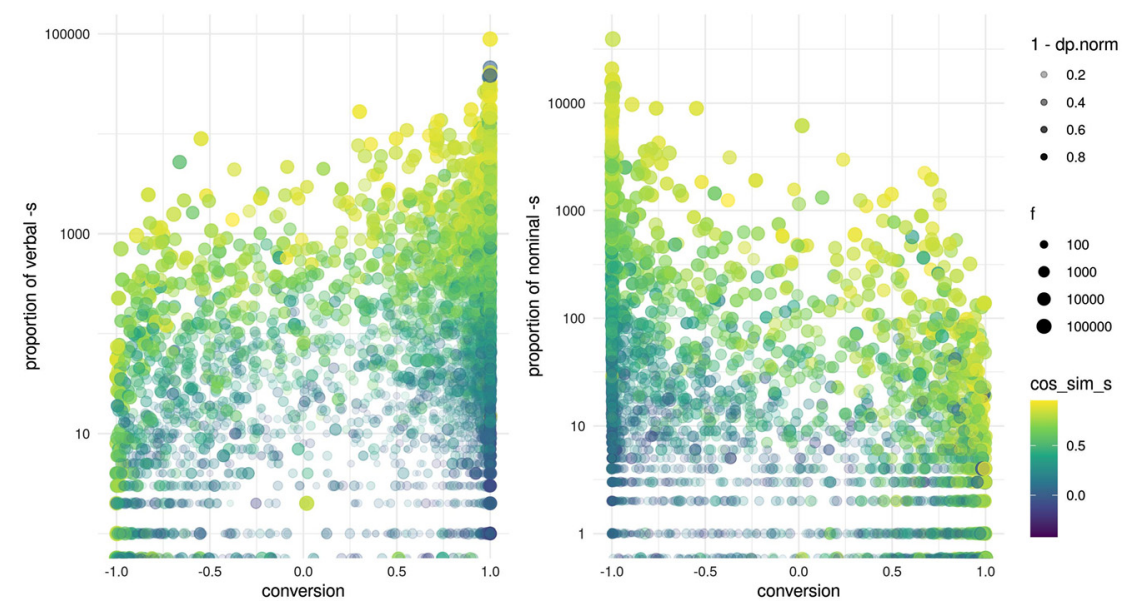

Figure 2: Compound ratio of noun/verb POS tags against occurrences of $\star_{-} s^{\star}$.

use context considerably, and in extreme cases, be indicative of highly conventionalized uses. Consider the following pairs:

(5) people, peoples (cosine similarity: 0.54)

(6) world, worlds (cosine similarity: 0.55)

(7) absence, absences (cosine similarity: 0.55)

Compared to:

(8) word, words (cosine similarity: 0.90)

(9) year, years (cosine similarity: 0.92)

(10) suit, suits (cosine similarity: 0.92)

In $5-7$, the most common singular use is semantically very different from the most common plural use, while in 8-10 there is no substantial semantic difference between the singular and the plural or across the different polysemes/homonyms of the pairs.

Even though the measure is correlated with frequency, it shows little correlation with the other measures listed above, except for DP.norm. 


\section{Analysis}

\subsection{Modeling Inflection Ratio}

In the following sections, three different regression models will be presented. Their purpose is mostly a first exploration of the above-mentioned measures and their influence on inflection across the verb-noun continuum. The relationship between the variables can be expected to be non-monotonic and non-linear. To allow for the necessary flexibility in the distribution and fit, the model framework chosen is GAMLSS (Rigby and Stasinopoulos 2005). It is a semi-parametric approach that allows us to fit a wide variety of distributions, and combine linear terms with smoothed terms. The models were created with the R package gamlss (Stasinopoulos et al. 2017).

The independent variable to be modeled was chosen to be the ratio of inflected forms relative to all occurrences of a lemma that were tagged as the compatible word class, which is noun for nominal $-s$ and verb for verbal $-s$ and -ed. Human perception has been found to be more sensitive to proportional changes of stimuli rather than absolute ones (cf. Kromer 2003). This makes counts of inflected forms an inherently upper-bounded, compositional phenomenon. The possible values can range from 0 to 1 . Therefore, the distribution chosen to be fitted was a zero-oneinflated beta distribution (Ospina and Ferrari 2012; Rigby et al. 2019). Raw counts of -s could not be successfully modeled directly using Poisson or negative (beta-) binomial regression, which led to highly problematic model properties, and a very poor model fit, or outright failure of the algorithm altogether.

Plain, relative or log frequencies did not improve the models, and exhibited inferior performance compared to all other metrics. In fact, model diagnostics became much worse in some cases. Possible reasons are the problematic distributional properties of word frequencies, and low frequency noise. Furthermore, frequency influences every of the remaining metrics, so it is in a sense heavily encoded there. Therefore, it was discarded in the final models presented here. DP.norm behaved in a very similar way, which is not surprising since it correlates strongly with frequency. Furthermore, it also correlates moderately with the other measures, making it a kind of in-between measure. It was also removed from the final models.

The above-mentioned measures, DWG, DP.norm (Section 3.1), alpha $a_{1}$ for both left and right neighbors (Section 3.2), and the conversion ratio introduced in (Section 2.3) where finally picked as predictors. The first regression model attempts to describe verbal uses of $-s$ based on parts-of-speech tagging (POS) tagging. The 
second and third model repeat the same procedure for-ed, both as past tense and past participle, and nominal -s.

\subsection{Data}

The data used to train the models was taken from the BNC (The British National Corpus 2007). The basic unit of analysis is lemmatized tokens, in combination with the CLAWS POS tags. It has to be noted that conversion is not necessarily restricted to single word units, but also possible for multi-word units or entire phrases, in the same way that such units can be lexicalized and stored as a whole. To keep the complexity of this study at a manageable level, only lexemes represented by individual words are considered. All metrics were calculated on all occurrences of a lemma rather than the tuples of lemma and POS tag as it is typically done. In this way, it is possible to capture a wider range of statistics per item, while also minimizing any a priori categorization of items, acknowledging the fact that lexical items in English are rather flexible. The default assumption is that sameness in form has a strong potential for lexical association between different uses. Of course, this assumption is considerably weakened in the case of homographs. A reliable method to annotate homographs, therefore, would be desirable.

Deverbal adjectives were filtered out based on POS tagging. Since the measures used as dependent and independent variables are all inherently ratio based, they are undefined at 0 occurrences of their significant category. Therefore, the dataset had to be split into two separate training sets, each with lemmas that occurred at least once as a verb (for the verbal -s and -ed models), or once as a noun (for the nominal $-s$ models). This explains the difference in sample sizes (cf. 7.1). A heuristic cut-off point of 50 occurrences was chosen since all metrics are increasingly subject to quantization effects and become rather unstable in lower frequency bands. Finally, proper names were commonly mistagged as verbs in the datasets, causing heavy tails in the residuals, and were therefore excluded from the final analysis.

\subsection{Model Fit}

Table 1 shows the statistics for the residual distribution Means and variances are very close to the desired values. The model for verbal $-s$ also shows a heavy right tail, which is caused by a high amount of extreme values. Arguably, this is not unusual for language data, and might be related to the sample size. All models show some degree of skew. An inherent property of the dataset is a potential for 
Table 1: Statistics for residuals and fit of the three GAMLSS models.

\begin{tabular}{lrrr}
\hline Metric & Verbal $-s$ & Nominal $-s$ & -ed \\
\hline Mean & -0.0382 & -0.0361 & 0.0261 \\
Variance & 0.9816 & 0.9227 & 0.9043 \\
Coef. of skewness & 0.5567 & 0.4772 & -0.2103 \\
Coef. of kurtosis & 5.7153 & 3.1176 & 3.6533 \\
Filliben correlation coefficient & 0.9894 & 0.9916 & 0.9955 \\
Pseudo $R^{2}$ & 0.6532 & 0.3914 & 0.3836 \\
\hline
\end{tabular}

multi-modality since the basic unit of analysis is lemmas. Clustering techniques or fitting model mixes might be strategies for improvement, without making arbitrary assumptions on lexical classes and creating cut-off points, e.g. for dominantly nominal versus dominantly verbal lexemes.

The overall deviance explained by the models is rather high for the verbal $-s$ model and medium for the other two models. There is much more variation on the nominal side of the spectrum, hence more variation when it comes to plural -s, which is also the most frequent inflection in comparison. However, the predictions of the model have to be taken with a grain of salt, since the distributional properties of the data could not be fitted perfectly, resulting in skewed residuals. Some potential factors could be the non-randomness of the data, strong systematic noise, such as homography, and potential unaccounted multimodality, e.g. caused by other word classes. Nevertheless, the applied dispersion and specificity measures allowed to improve the fit drastically, and show promise for future improvements.

The full summary of the models including estimates, $p$-values and confidence intervals for every coefficient can be found in the appendix, Section A.1, residual plots in Section A.3.

\subsection{Influence of Conversion on Nominal and Verbal -s versus -ed}

Figure 3 shows a side by side comparison of the estimates for conversion. The effect of conversion on nominal $-s$ follows a distinct U-shape with both ends of the continuum having little to no effect. Figure $3 \mathrm{~b}$ shows a less pronounced U-shape, and a much larger positive effect on the nominal side of the spectrum. In both cases, there is a depression towards the middle of the continuum rather than a simple monotonic relationship.

In comparison, the influence of conversion on -ed is similar, but much smaller and prone to more uncertainty as can be seen in Figure 3c. 


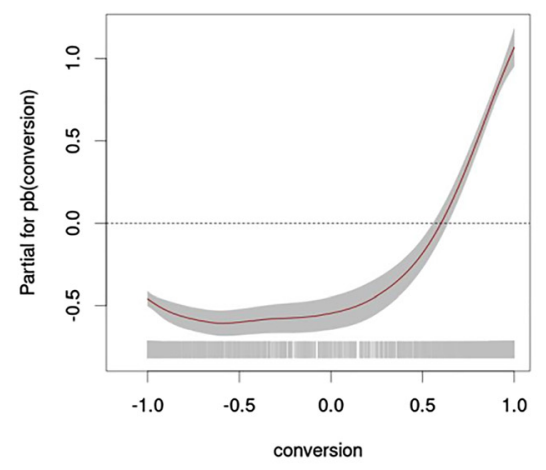

(a) verbal $-s$

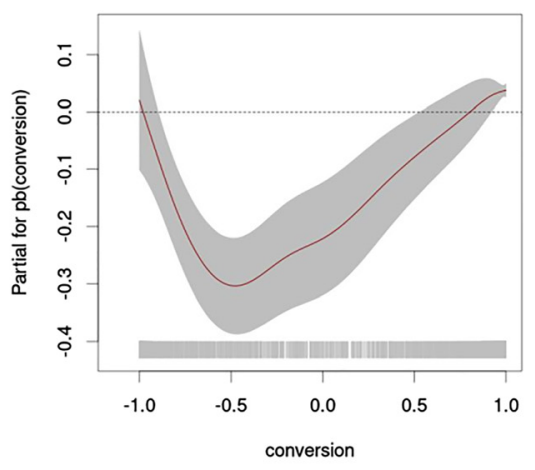

(b) nominal $-s$

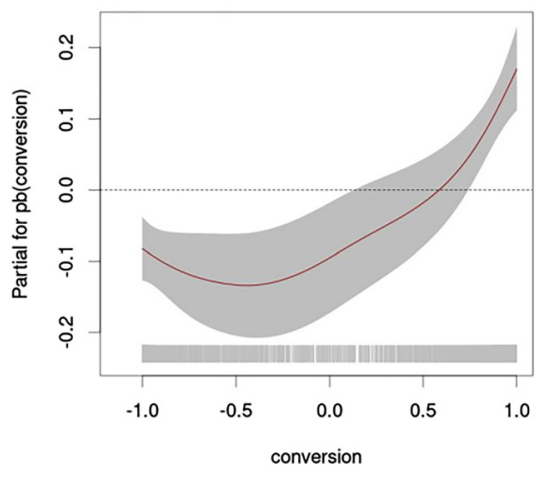

(c) $-e d$

Figure 3: Model estimates for conversion compared across the three models.

\subsection{Influence of Dispersion and Fixedness in Context}

Figure 4 shows the remaining coefficients for the verbal -s model. ${ }^{3}$ The effect of $D W G$ was only significant in the model for nominal $-s$ (see Section A.1). There, it showed the expected effect: a sharp increase of probability for inflection at high values. Aside from that, controlling for $D W G$ did improve the distribution of residuals, and $D W G$ had a significant and comparatively large effect on the scale parameter in the verbal $-s$ model, indicating that it could successfully account for some of the heteroscedasticity

3 When only one set of graphs is shown, the patterns depicted are roughly representative of those observed for the other two models unless further discussed. 

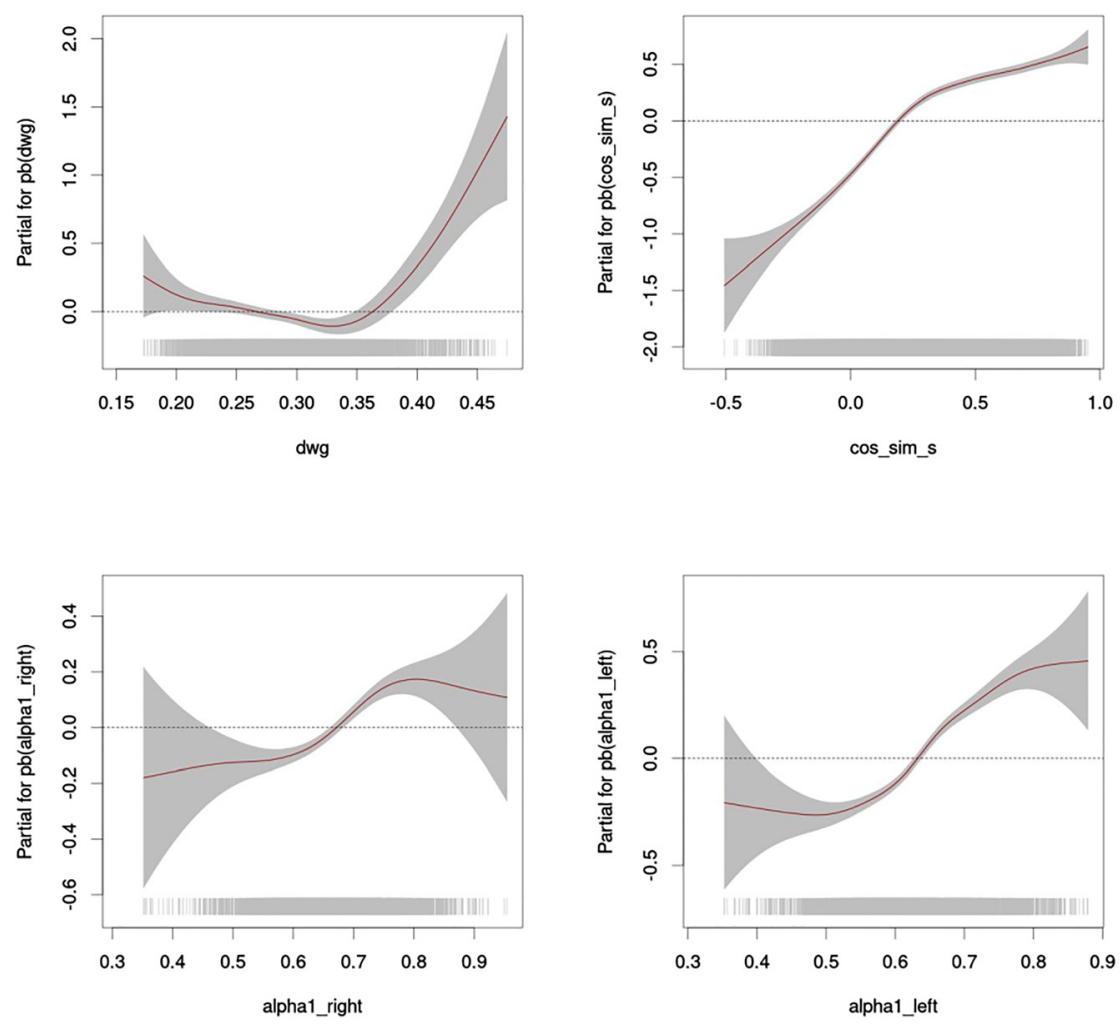

Figure 4: Model estimates for conversion compared across the three models.

in the distribution. The variation is higher in both extreme ends of the measure. Interestingly, very highly dispersed lexemes show the most variable behavior.

The boundedness to left- and right-hand-side tokens measured by alph $a_{1}$ shows a slightly positive effect for extremely flexible lemmas, and a negative one for extremely inflexible ones. This pattern holds across all models and is only subject to larger fluctuations at the extreme ends. The largest slope can be observed in the left context in the model for nominal $-s$ and the right context of verbal $-s$ (cf. Section A.1). In the case of nouns, the left context is where immediate morpho-syntactic markers occur, such as determiners. This pattern could be an indication that inflected cases of conversion are morpho-syntactically more restricted.

Finally, the cosine similarities from the GloVe embeddings show the most distinct positive slope. A similarity in lexical co-occurrence patterns between the base and its inflected form is correlated with a higher proportion of occurrences of the inflectional form. 


\subsection{Zero Components of the Model}

Inflection is not semantically or functionally viable for all lexemes, therefore there is a high amount of items that never occur inflected. As compound models, the coefficients have different values for this zero part of the distribution. Figure 5 shows the influence of conversion and cosine similarity on the probability that a lexeme has an inflection ratio of 0 . The end points show a consistently high/low influence towards the respective parts of the continuum. For verbs, the probability to never occur with a verbal -s or -ed is lowest when there is no conversion. As soon as there is some degree of conversion this drastically changes. In the middle of the continuum, the effect flattens until there is a sharp rise for items that have high proportions of nominal uses, meaning nouns with few verbal conversions are more likely to occur with verbal inflection. The model for nominal $-s$ mirrors this trend.

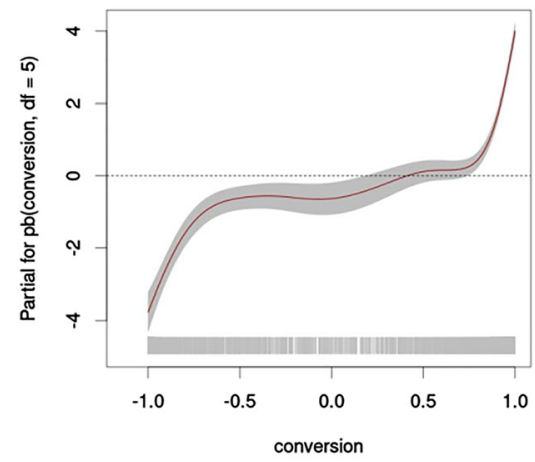

(a) verbal $-s$

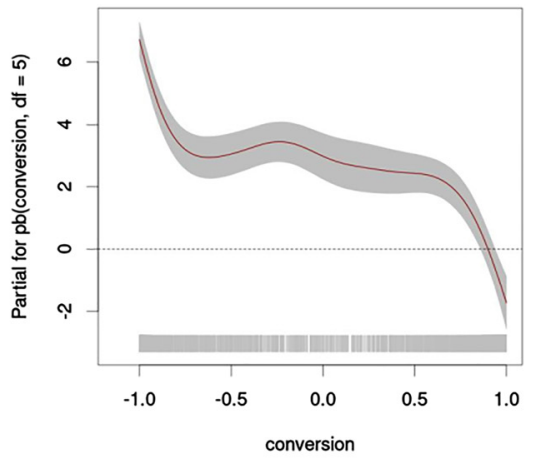

(b) nominal $-s$

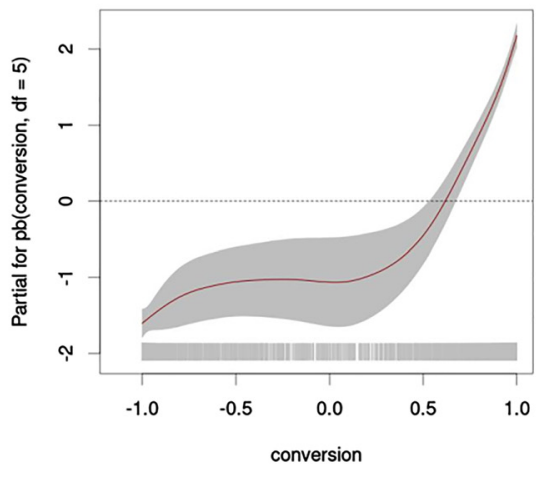

(c) $-e d$

Figure 5: Model estimates for conversion compared across the three models. 
The 1 components of the models were mostly inconclusive, and therefore not visualized here. The reason for that lies in the extremely low amount of items that were always inflected. Most of these items had extreme values due to tagging or lemmatization errors. The few legitimate observations had very low frequencies. For nominal $-s$, pluralia tantum are a candidate category that has the potential to form a cluster, but either it is not a productive category or the sample size was just too low or the data too noisy to really see it. Furthermore, even most pluralia tantum will eventually be analogically backformed or otherwise used in a singular form at least once given a sufficiently large sample size. Theoretically, verbal -s has no plausible category analogous with pluralia tantum, neither does -ed. Dropping the 1-inflated component and using a correction for ratios equal to 1 appears to be a valid alternative strategy this type of model.

\section{Discussion}

Modeling the proportion of -s occurrences showed a generally negative effect for items that are word class ambiguous. The probability of occurrence with inflection sharply decreases towards the "uncanny valley" of conversion. This decrease is more pronounced at the nominal side of the continuum. Interestingly, however, the probability of inflection seems to rise again towards the opposite side for all three inflectional suffixes. In the case of verb-noun conversion, this means that inflectional marking is more common than expected for verbs that are not also used as nouns and therefore more strongly entrenched as verbs than their more flexible counterparts toward the middle of the continuum. The same can be said about the noun-verb conversion. The absence of additional morphological cues might contribute to the difficulty in processing. This is somewhat counter-balanced by higher inflection ratios for very flexible collocates indicated by a positive effect of the $a l p h a_{1}$ and $D W G$ values, i.e. higher inflection ratio with highly flexible, and well-dispersed uses.

Moreover, a distinct U-shape can be observed for the effect of conversion on nominal -s. This suggests that verbs are as likely to be pluralized when they are adhoc conversions or have rather rare nominal homonyms/polysemes. The U-shape is less pronounced but still noticeable in the other models. It is possible that a lack of entrenchment as nominal/verbal lexeme, which potentially makes inflection a bit more awkward, is counter-acted by other patterns. Additionally, the probability that items never occur with the -s suffix of their respective dominant word class was shown to drastically decrease already at rather low proportions of conversion. The lack of morphological marking for word classes was expected to require additional morpho-syntactic marking if the word class association is blurred, since additional marking can increase the ease of processing. The results, however, suggest that other distributional properties play a much larger role. 


\section{Conclusion and Outlook}

This study analyzed the underlying distribution of inflectional suffixes across the verb-noun continuum, in order to trace effects of word class ambiguity. There is a lot of structure in corpus data, and the multivariate approach presented here shows a high potential for identifying trends otherwise drowned by noisy data or covered by highly skewed overlapping distributions. Additionally, understanding morphological data as proportional, rather than count data, allows for intuitive and conceptually interesting interpretations. The observations in the corpus data are in line with previous research in phonetics and neurolinguistic.

Measures of lexical fixedness/productivity more sophisticated than the proposed fixed-window hapax ratio are also desirable, especially to capture constructions, constructional idioms and other semi-fixed structures. In fact, careful application and improvements on the entire stack of corpus analysis are required, all the way from tokenization, over lemmatization, to POS tagging. In future studies, customized procedures have to be considered that are able to denoise the information required for a problem like ambiguity, rather than relying on premade one-size-fits-all solutions. This comes at a considerable computation effort but is becoming more and more feasible with modern hardware and/or distributed systems.

Word embeddings, dispersion measures and association statistics show that individual word statistics work best when taking into account the entire corpus. Word embeddings and more recent transformer networks like BERT may provide an interesting route for further studies in ambiguity (e.g. Beekhuizen, Armstrong, and Stevenson 2021; Du, Qi, and Sun 2019; Wiedemann et al. 2019). Clustering techniques can be used to detect homonymy (Lee 2021), which could be used for corpus annotation as an addition to or replacement of classical lemmatization. If proven robust and carefully applied, this could potentially lead to further decreases in noise. The recent successes of word2vec, BERT etc. in practical application are promising for the use in a more descriptive application. They can provide another angle on co-occurrence statistics, and were only sparsely used in this study since there has been little systematic application in corpus linguistics prior to this point. The mentioned techniques can be further enriched by including more contextual and "world knowledge” information, such as images (e.g. Kottur et al. 2016; Shahmohammadi, Lensch, and Baayen 2021). For the time being, more well-understood dispersion measures, measures of productivity and simple context embeddings can still provide tools to further test where real ambiguity exists and how it affects the system of language. 


\section{A Appendix}

\section{A.1 Model Summaries}

\section{A.1.1 Verbal -s}

\begin{tabular}{|c|c|c|c|c|c|}
\hline Parameter & Coefficient & SE & $95 \% \mathrm{CI}$ & $t(5810.18)$ & $p$ \\
\hline \multicolumn{6}{|l|}{ \# Fixed effects } \\
\hline (Intercept) & -4.79 & 0.24 & {$[-5.26,-4.33]$} & -20.20 & $<0.001$ \\
\hline conversion & 0.56 & 0.02 & {$[0.51,0.60]$} & 24.16 & $<0.001$ \\
\hline dwg & 0.07 & 0.37 & {$[-0.66,0.81]$} & 0.20 & 0.841 \\
\hline cos_sim_s & 0.90 & 0.05 & {$[0.79,1.00]$} & 16.73 & $<0.001$ \\
\hline alpha1_right & 1.89 & 0.23 & {$[1.45,2.34]$} & 8.28 & $<0.001$ \\
\hline alpha1_left & 1.47 & 0.20 & {$[1.09,1.86]$} & 7.53 & $<0.001$ \\
\hline \multicolumn{6}{|l|}{ \# Sigma } \\
\hline (Intercept) & -3.15 & 0.24 & {$[-3.62,-2.68]$} & -13.25 & $<0.001$ \\
\hline $\mathrm{pb}($ conversion, $d f=5)$ & 0.64 & 0.02 & {$[0.60,0.68]$} & 30.04 & $<0.001$ \\
\hline dwg & 2.05 & 0.36 & {$[1.34,2.76]$} & 5.66 & $<0.001$ \\
\hline alpha1_right & 1.16 & 0.24 & {$[0.69,1.62]$} & 4.86 & $<0.001$ \\
\hline alpha1_left & 1.24 & 0.21 & {$[0.84,1.65]$} & 5.97 & $<0.001$ \\
\hline cos_sim_s & 0.27 & 0.05 & {$[0.16,0.37]$} & 4.90 & $<0.001$ \\
\hline \multicolumn{6}{|l|}{$\# \mathrm{Nu}$} \\
\hline (Intercept) & -2.47 & 0.73 & {$[-3.89,-1.05]$} & -3.41 & $<0.001$ \\
\hline $\mathrm{pb}($ conversion, $d f=5)$ & 2.85 & 0.09 & {$[2.67,3.03]$} & 31.07 & $<0.001$ \\
\hline dwg & -0.02 & 0.96 & {$[-1.91,1.87]$} & -0.02 & 0.982 \\
\hline alpha1_right & 0.47 & 0.72 & {$[-0.93,1.88]$} & 0.66 & 0.511 \\
\hline alpha1_left & 1.39 & 0.69 & {$[0.03,2.74]$} & 2.01 & 0.045 \\
\hline cos_sim_s & -2.16 & 0.17 & {$[-2.49,-1.83]$} & -12.84 & $<0.001$ \\
\hline \multicolumn{6}{|l|}{ \# Tau } \\
\hline (Intercept) & -14.11 & 3.06 & {$[-20.10,-8.12]$} & -4.62 & $<0.001$ \\
\hline $\mathrm{pb}($ conversion,$d f=5)$ & 4.92 & 2.01 & {$[0.98,8.85]$} & 2.45 & 0.014 \\
\hline dwg & -1.83 & 2.52 & {$[-6.77,3.10]$} & -0.73 & 0.467 \\
\hline alpha1_right & 1.96 & 1.88 & {$[-1.73,5.66]$} & 1.04 & 0.298 \\
\hline alpha1_left & 9.45 & 2.68 & {$[4.20,14.71]$} & 3.52 & $<0.001$ \\
\hline cos_sim_s & -2.13 & 0.64 & {$[-3.38,-0.88]$} & -3.33 & $<0.001$ \\
\hline No. of observations in the fit: & & & & & 5923 \\
\hline Degrees of freedom for the fit: & & & & & 12.8151 \\
\hline Residual deg. of freedom: & & & & & 810.185 \\
\hline At cycle: & & & & & 30 \\
\hline Global deviance: & & & & & 669.721 \\
\hline AIC: & & & & & 444.091 \\
\hline SBC: & & & & & 689.741 \\
\hline
\end{tabular}




\section{A.1.2 Nominal -s}

\begin{tabular}{|c|c|c|c|c|c|}
\hline Parameter & Coefficient & SE & $95 \% \mathrm{CI}$ & $t(12117.43)$ & $p$ \\
\hline \multicolumn{6}{|l|}{ \# Fixed effects } \\
\hline (Intercept) & -4.61 & 0.15 & {$[-4.91,-4.31]$} & -29.87 & $<0.001$ \\
\hline conversion & 0.12 & 0.02 & {$[0.08,0.17]$} & 6.11 & $<0.001$ \\
\hline dwg & 1.35 & 0.20 & {$[0.96,1.73]$} & 6.77 & $<0.001$ \\
\hline cos_sim_s & 1.37 & 0.04 & {$[1.30,1.45]$} & 36.57 & $<0.001$ \\
\hline alpha1_right & 1.58 & 0.16 & {$[1.28,1.89]$} & 10.22 & $<0.001$ \\
\hline alpha1_left & 2.68 & 0.15 & {$[2.39,2.97]$} & 18.36 & $<0.001$ \\
\hline \multicolumn{6}{|l|}{ \# Sigma } \\
\hline (Intercept) & 0.55 & 0.14 & {$[0.28,0.83]$} & 3.91 & $<0.001$ \\
\hline $\mathrm{pb}($ conversion, $d f=5)$ & 0.03 & 0.02 & {$[-0.01,0.07]$} & 1.45 & 0.146 \\
\hline dwg & 0.24 & 0.18 & {$[-0.12,0.60]$} & 1.31 & 0.190 \\
\hline alpha1_right & -0.83 & 0.14 & {$[-1.11,-0.55]$} & -5.85 & $<0.001$ \\
\hline alpha1_left & 0.09 & 0.13 & {$[-0.17,0.36]$} & 0.70 & 0.485 \\
\hline cos_sim_s & -0.91 & 0.04 & {$[-0.98,-0.84]$} & -25.15 & $<0.001$ \\
\hline \multicolumn{6}{|l|}{$\# \mathrm{Nu}$} \\
\hline (Intercept) & -1.06 & 1.28 & {$[-3.57,1.45]$} & -0.83 & 0.408 \\
\hline $\mathrm{pb}($ conversion, $d f=5)$ & -2.96 & 0.16 & {$[-3.27,-2.66]$} & -18.98 & $<0.001$ \\
\hline dwg & -5.05 & 2.05 & {$[-9.06,-1.04]$} & -2.47 & 0.014 \\
\hline alpha1_right & -2.72 & 1.21 & {$[-5.10,-0.35]$} & -2.25 & 0.025 \\
\hline alpha1_left & 1.24 & 0.97 & {$[-0.66,3.15]$} & 1.28 & 0.201 \\
\hline cos_sim_s & -1.90 & 0.31 & {$[-2.50,-1.30]$} & -6.19 & $<0.001$ \\
\hline \multicolumn{6}{|l|}{ \# Tau } \\
\hline (Intercept) & -8.92 & 1.40 & {$[-11.67,-6.17]$} & -6.36 & $<0.001$ \\
\hline $\mathrm{pb}($ conversion,$d f=5)$ & -1.00 & 0.12 & {$[-1.23,-0.77]$} & -8.46 & $<0.001$ \\
\hline dwg & 4.66 & 1.75 & {$[1.24,8.09]$} & 2.67 & 0.008 \\
\hline alpha1_right & -0.47 & 1.35 & {$[-3.12,2.18]$} & -0.35 & 0.726 \\
\hline alpha1_left & 5.64 & 1.30 & {$[3.10,8.18]$} & 4.35 & $<0.001$ \\
\hline cos_sim_s & -1.06 & 0.40 & {$[-1.84,-0.27]$} & -2.63 & 0.009 \\
\hline No. of observations in the fit: & & & & & 12198 \\
\hline Degrees of freedom for the fit: & & & & & 0.57188 \\
\hline Residual deg. of freedom: & & & & & 2117.43 \\
\hline At cycle: & & & & & 14 \\
\hline Global deviance: & & & & & 0043.74 \\
\hline AIC: & & & & & -9882.6 \\
\hline SBC: & & & & & 285.641 \\
\hline
\end{tabular}




\section{A.1.3 -ed}

\begin{tabular}{|c|c|c|c|c|c|}
\hline Parameter & Coefficient & SE & $95 \% \mathrm{CI}$ & $t(5861.88)$ & $p$ \\
\hline \multicolumn{6}{|l|}{ \# Fixed effects } \\
\hline (Intercept) & -1.60 & 0.24 & {$[-2.08,-1.12]$} & -6.57 & $<0.001$ \\
\hline conversion & 0.11 & 0.02 & {$[0.07,0.14]$} & 5.41 & $<0.001$ \\
\hline dwg & 0.16 & 0.39 & {$[-0.59,0.92]$} & 0.42 & 0.673 \\
\hline alpha1_right & 1.85 & 0.23 & {$[1.39,2.31]$} & 7.92 & $<0.001$ \\
\hline alpha1_left & 0.16 & 0.20 & {$[-0.24,0.56]$} & 0.79 & 0.429 \\
\hline \multicolumn{6}{|l|}{ \# Sigma } \\
\hline (Intercept) & 1.09 & 0.23 & {$[0.65,1.54]$} & 4.79 & $<0.001$ \\
\hline $\mathrm{pb}($ conversion, $d f=5)$ & 0.02 & 0.02 & {$[-0.02,0.06]$} & 1.04 & 0.301 \\
\hline dwg & 0.14 & 0.37 & {$[-0.58,0.85]$} & 0.37 & 0.709 \\
\hline alpha1_right & -1.40 & 0.23 & {$[-1.84,-0.95]$} & -6.10 & $<0.001$ \\
\hline alpha1_left & -0.55 & 0.20 & {$[-0.96,-0.15]$} & -2.70 & 0.007 \\
\hline \multicolumn{6}{|l|}{$\# \mathrm{Nu}$} \\
\hline (Intercept) & -9.45 & 0.64 & {$[-10.71,-8.18]$} & -14.65 & $<0.001$ \\
\hline $\mathrm{pb}($ conversion, $d f=5)$ & 1.70 & 0.07 & {$[1.58,1.83]$} & 25.84 & $<0.001$ \\
\hline dwg & 2.93 & 0.90 & {$[1.15,4.70]$} & 3.23 & 0.001 \\
\hline alpha1_right & 2.98 & 0.64 & {$[1.73,4.23]$} & 4.68 & $<0.001$ \\
\hline alpha1_left & 7.24 & 0.62 & {$[6.03,8.46]$} & 11.69 & $<0.001$ \\
\hline \multicolumn{6}{|l|}{ \# Tau } \\
\hline (Intercept) & -10.75 & 1.09 & {$[-12.89,-8.62]$} & -9.87 & $<0.001$ \\
\hline $\mathrm{pb}($ conversion,$d f=5)$ & 3.49 & 0.37 & {$[2.77,4.22]$} & 9.44 & $<0.001$ \\
\hline dwg & 1.81 & 1.49 & {$[-1.11,4.73]$} & 1.21 & 0.225 \\
\hline alpha1_right & 2.13 & 1.10 & {$[-0.03,4.29]$} & 1.93 & 0.054 \\
\hline alpha1_left & 6.14 & 1.08 & {$[4.02,8.27]$} & 5.67 & $<0.001$ \\
\hline No. of observations in the fit: & & & & & 5923 \\
\hline Degrees of freedom for the fit: & & & & & 61.11825 \\
\hline Residual deg. of freedom: & & & & & 5861.882 \\
\hline At cycle: & & & & & 8 \\
\hline Global deviance: & & & & & 4316.905 \\
\hline AIC: & & & & & 4439.142 \\
\hline SBC: & & & & & 4847.815 \\
\hline
\end{tabular}

\section{A.2 Nomenclature}

All models are zero-one-inflated beta regressions with the proportion of inflection as dependent variable. Sigma coefficients represent the scale parameter of the distribution, $\mathrm{Nu}$ and Tau represent the 0 and 1 component respectively. All coefficients were fitted using p-splines. Non-fixed effects were set to $d f=5$ to control for overfitting. The 
mean and scale parameter use the pre-specified logit link function. $\mathrm{Nu}$ and Tau have $\log$ link functions.

\section{A.3 Residual Plots}
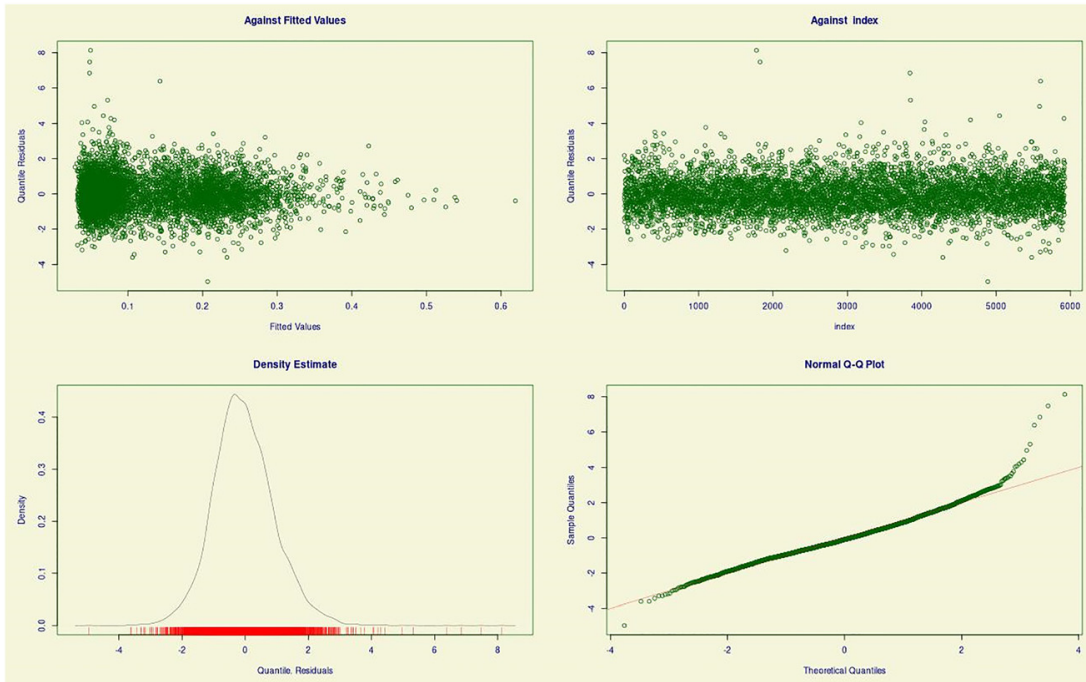

Figure 6: Diagnostics for beta inflated GAMLSS model predicting proportions of verbal -s.
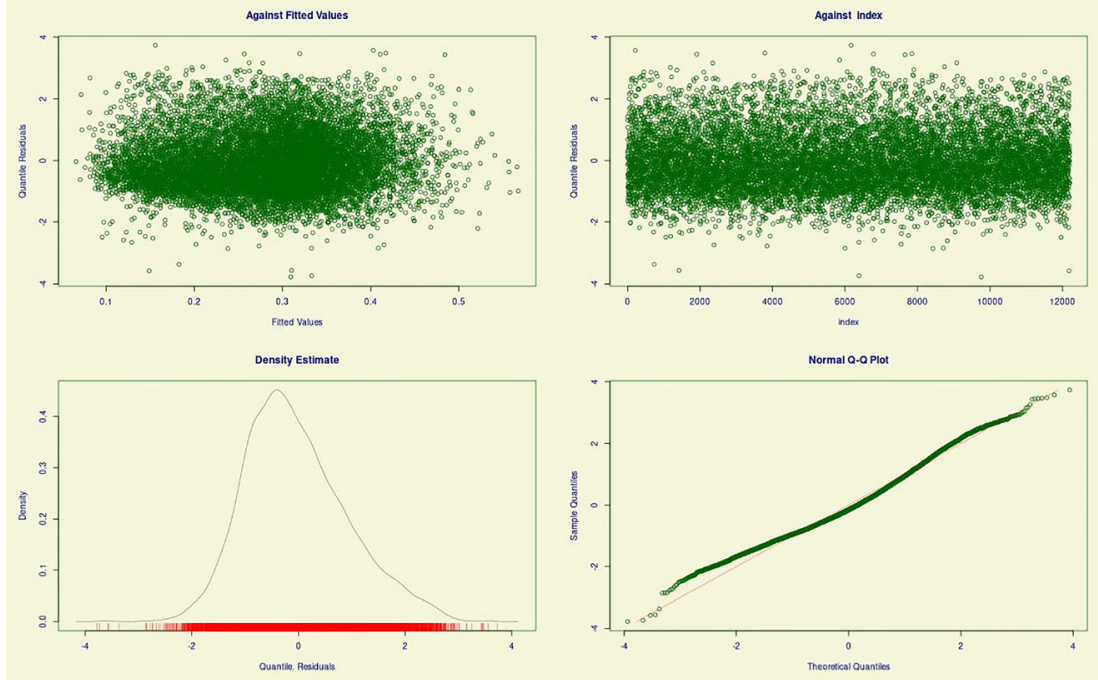

Figure 7: Diagnostics for beta inflated GAMLSS model predicting proportions of nominal -s. 

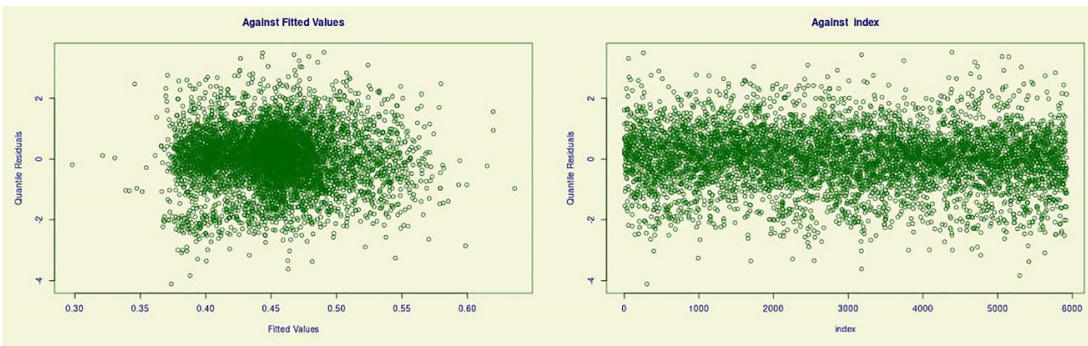

Density Estimate

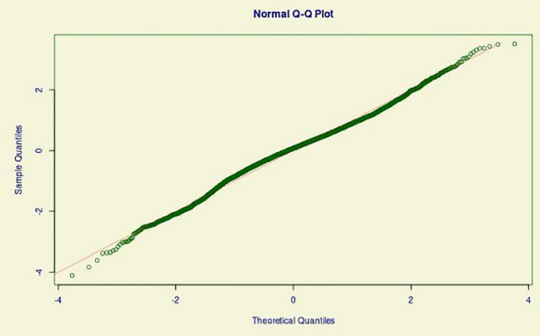

Figure 8: Diagnostics for beta inflated GAMLSS model predicting proportions of -ed.

\section{References}

Beekhuizen, B., Armstrong, B. C., and Stevenson, S. (2021). Probing Lexical Ambiguity: Word Vectors Encode Number and Relatedness of Senses. Cognitive Science 45: 5.

Bultena, S., Dijkstra, T., and van Hell, J. G. (2013). Cognate and Word Class Ambiguity Effects in Noun and Verb Processing. Language \& Cognitive Processes 28(9): 1350-77.

Diessel, H. (2016). Frequency and Lexical Specificity in Grammar: A Critical Review. In: Heike, B., and Pfänder, S. (Eds.), Experience Counts: Frequency Effects in Language. De Gruyter, Berlin, pp. 209-38.

Dowle, M., and A. Srinivasan. 2021. Data.table: Extension of 'Data.frame'. Also available at https://CRAN.R-project.org/package=data.table.

Du, J., F. Qi, and M. Sun. 2019. Using BERT for Word Sense Disambiguation. CoRR abs/1909.08358. Also available at http://arxiv.org/abs/1909.08358.

Evert, S. 2005. "The Statistics of Word Cooccurrences: Word Pairs and Collocations." PhD thesis, Friedrich-Alexander-Universität Erlangen-Nürnberg. http://www.collocations.de/phd.html.

Evert, S., and Hardie, A. (2011). Twenty-First Century Corpus Workbench: Updating a Query Architecture for the New Millennium. University of Birmingham, Birmingham.

Federmeier, K. D., Segal, J. B., Lombrozo, T., and Kutas, M. (2000). Brain Responses to Nouns, Verbs and Class-Ambiguous Words in Context. Brain 123(12): 2552-66.

Gries, S. (2021). A New Approach to (Key) Keywords Analysis: Using Frequency, and Now Also Dispersion. Research in Corpus Linguistics 9(2): 1-33.

Gries, S. T. 2008. “Dispersions and Adjusted Frequencies in Corpora." International Journal of Corpus Linguistics 13 (4): 403-37. 
Kottur, S., R. Vedantam, M. F. M. José, and D. Parikh. 2016. "Visual Word2vec (vis-w2v): Learning Visually Grounded Word Embeddings Using Abstract Scenes." In Proceedings of the IEEE Conference on Computer Vision and Pattern Recognition, 4985-94. San Francisco: IEEE.

Kromer, V. 2003. "A Usage Measure Based on Psychophysical Relations." Journal of Quantitative Linguistics 10 (2): 177-86.

Lee, C. L., and Federmeier, K. D. (2006). To Mind the Mind: An Event-Related Potential Study of Word Class and Semantic Ambiguity. Brain Research 1081(1): 191-202.

Lee, C. L., and Federmeier, K. D. (2008). To Watch, to See, and to Differ: An Event-Related Potential Study of Concreteness Effects as a Function of Word Class and Lexical Ambiguity. Brain and Language 104(2): 145-58.

Lee, Y. (2021). Systematic Homonym Detection And Replacement Based On Contextual Word Embedding. Neural Processing Letters 53(1): 17-36.

Lijffijt, J., and S. T. Gries. 2012. "Correction to Stefan Th. Gries' "Dispersions and Adjusted Frequencies in Corpora”." International Journal of Corpus Linguistics 13 (4 (2008)): 403-37.

Ospina, R., and Ferrari, S. L. P. (2012). A General Class of Zero-or-One Inflated Beta Regression Models. Computational Statistics \& Data Analysis 56(6): 1609-23.

Pennington, J., R. Socher, and C. D. Manning. 2014. "Glove: Global Vectors for Word Representation." In Proceedings of the 2014 Conference on Empirical Methods in Natural Language Processing (EMNLP), 1532-43. Doha: Association for Computational Linguistics.

Piantadosi, S. T., Tily, H., and Gibson, E. (2012). The Communicative Function of Ambiguity in Language. Cognition 122(3): 280-91.

Plag, I., J. Homann, and G. Kunter. 2017. "Homophony and Morphology: The Acoustics of WordFinal S in English.” Journal of Linguistics 53 (1): 181-216.

R Core Team. 2021. R: A Language and Environment for Statistical Computing. Vienna, Austria: $\mathrm{R}$ Foundation for Statistical Computing.

Rigby, R. A., and D. M. Stasinopoulos. 2005. "Generalized Additive Models for Location, Scale and Shape (With Discussion)." Applied Statistics 54 (3): 507-54.

Rigby, R. A., D. S. Mikis, G. Z. Heller, and F. De Bastiani. 2019. Distributions for Modeling Location, Scale, and Shape: Using GAMLSS in r. Chapman: Hall/CRC.

Selivanov, D., M. Bickel, and Q. Wang. 2020. text2vec: Modern Text Mining Framework for $r$. Also available at https://CRAN.R-project.org/package=text2vec.

Shahmohammadi, H., H. Lensch, and R. Harald Baayen. 2021. Learning Zero-Shot Multifaceted Visually Grounded Word Embeddings via Multi-Task Training. arXiv preprint arXiv: 2104.07500.

Stasinopoulos, M. D., R. A. Rigby, and F. De Bastiani. 2018. "GAMLSS: A Distributional Regression Approach." Statistical Modelling 18 (3-4): 248-73.

Stasinopoulos, M. D., Rigby, R. A., Heller, G. Z., Voudouris, V., and De Bastiani, F. (2017). Flexible Regression and Smoothing: Using GAMLSS in r. CRC Press, Boca Raton.

The British National Corpus, version 3 (BNC XML Edition). 2007. Distributed by Bodleian Libraries, University of Oxford, on Behalf of the BNC Consortium. Also available at http://www.natcorp. ox.ac.uk/.

Tomaschek, F., Plag, I., Ernestus, M., and Harald Baayen, R. (2021). Phonetic Effects of Morphology and Context: Modeling the Duration of Word-Finals in English with Naïve Discriminative Learning. Journal of Linguistics 57(1): 123-61.

Tomaschek, F., Tucker, B. V., Fasiolo, M., and Harald Baayen, R. (2018). Practice Makes Perfect: The Consequences of Lexical Proficiency for Articulation. Linguistics Vanguard 4(s2): 20170018. 
Trott, S., and Bergen, B. (2020). Why do Human Languages have Homophones? Cognition 205: 104449.

Wasow, T. (2015). Ambiguity Avoidance is Overrated. In: Susanne, W. (Ed.), Ambiguity. De Gruyter, Berlin, pp. 29-48.

Wickham, H. 2016. ggplot2: Elegant Graphics for Data Analysis. New York: Springer-Verlag.

Wiedemann, G., Steffen Remus, A. Chawla, and C. Biemann. 2019. "Does BERT Make Any Sense? Interpretable Word Sense Disambiguation with Contextualized Embeddings.” arXiv preprint arXiv:1909.10430.

Yung Song, J., K. Demuth, K. Evans, and S. Shattuck-Hufnagel. 2013. "Durational Cues to Fricative Codas in 2-Year-Olds' American English: Voicing and Morphemic Factors." Journal of the Acoustical Society of America 133 (5): 2931-46.

Zimmermann, R. 2020. "Word Growth Dispersion-A Single Corpus Part Measure of Lexical Dispersion." In Paper Presented at ICAME41 Heidelberg. Also available at https://www. youtube.com/watch?v=k8etOvRcF4c. 